\title{
Examination of the quality of spinach leaves using hyperspectral imaging
}

\author{
Belén Diezma ，Lourdes Lleó , Jean Michel Roger , Ana Herrero-Langreo , \\ Loredana Lunadei , Margarita Ruiz-Altisent
}

\begin{abstract}
A B S T R A C T
The present research is focused on the application of hyperspectral images for the supervision of quality deterioration in ready to use leafy spinach during storage (Spinacia oleracea). Two sets of samples of packed leafy spinach were considered: (a) a first set of samples was stored at $20^{\circ} \mathrm{C}(\mathrm{E}-20)$ in order to accelerate the degradation process, and these samples were measured the day of reception in the laboratory and after 2 days of storage; (b) a second set of samples was kept at $10^{\circ} \mathrm{C}(\mathrm{E}-10)$, and the measurements were taken throughout storage, beginning the day of reception and repeating the acquisition of Images 3, 6 and 9 days later. Twenty leaves per test were analyzed. Hyperspectral images were acquired with a push-broom CCD camera equipped with a spectrograph VNIR ( $400-1000 \mathrm{~nm})$. Calibration set of spectra was extracted from E-20 samples, containing three classes of degradation: class A (optimal quality), class B and class $C$ (maximum deterioration). Reference average spectra were defined for each class. Three models, computed on the calibration set, with a decreasing degree of complexity were compared, according to their ability for segregating leaves at different quality stages (fresh, with incipient and non-visible symptoms of degradation, and degraded): spectral angle mapper distance (SAM), partial least squares discriminant analysis models (PLS-DA), and a non linear index (Leafy Vegetable Evolution, LEVE) combining five wavelengths were included among the previously selected by CovSel procedure. In sets E-10 and E-20, artificial images of the membership degree according to the distance of each pixel to the reference classes, were computed assigning each pixel to the closest reference class. The three methods were able to show the degradation of the leaves with storage time.
\end{abstract}

\section{Introduction}

Fresh-cut fruit and vegetables, initially called minimally processed or lightly processed products, can be defined as any fresh fruit or vegetable that has been physically modified from its original state (by peeling, trimming, washing and cutting) to obtain a $100 \%$ edible product that is subsequently bagged or prepackaged and kept in refrigerated storage (Martín-Belloso and Soliva-Fortuny, 2011).

The sector is asking for innovative, fast, cheap and objective techniques to evaluate the overall quality and safety (or some of the specific quality parameters) of fresh-cut products in order to obtain decision tools for implementing new packaging procedures. Hyperspectral imaging technique could be a first approach. Such a system allows for integrating spectroscopic and imaging techniques to enable direct identification of different components or quality characteristics and their spatial distribution in the tested sample (ElMasry and Sun, 2010). Measurement of the optical properties of food products has been one of the most successful nondestructive techniques for quality assessment to provide several quality details simultaneously. In these spectroscopic techniques, it is possible to obtain information about the sample components based on the light absorption of the sample, but it is not easy to extract the information on position/location. Because hyperspectral imaging techniques overcome the limits of spectroscopic techniques and vision techniques, they have emerged as a powerful technique in agricultural and food systems. Based on hyperspectral imaging techniques, a multispectral imaging system can be built for real-time implementations (Lee et al., 2005). It involves measuring the intensity of diffusely reflected light from a surface at one or more wavelengths with relatively narrow band passes. Since image data are considered two-dimensional, by adding a new dimension of "spectrum" information, the hyperspectral image data can be perceived as a three-dimensional data cube (Chao et al., 2001). Hyperspectral imaging, like other spectroscopy techniques, can be carried out in reflectance, transmission or fluorescence modes, reflectance being the most usual mode. 
One of the main challenges in hyperspectral vision is the management and analysis of large and complex databases to extract relevant information contained in them (Fernandez Pierna et al., 2010). The starting points for this are the methods of spectral preprocessing (normalization, smoothing, differentiation, etc.) and multivariate analysis (correlation techniques, principal component analysis, discriminant analysis, etc.) traditionally applied to spectroscopy (Gowen et al., 2007). In the case of hyperspectral vision, these procedures can be applied to the whole image or to sub-populations of pixels representative of the variability of the samples. The projection of the whole images onto new spaces generated by multivariate analysis or the computation of indexes based on some wavelengths generate virtual images that must be analyzed searching for similarity.

With hyperspectral imaging, spectral pre-processing techniques are applied to remove non-chemical biases from the spectral information, e.g. scattering, temperature influence (HernandezSanchez et al., 2003) or device calibrations (Gowen et al., 2007; Fearn et al., 2009; Rinnan et al., 2009). Geometric pre-processing methods are applied to correct spectral data from drift baseline, non linearity, curvilinearity, as well as additive and multiplicative effects. Some of these methods are the standard normal variate transform (SNV), smoothing and differentiation (Zeaiter et al., 2005). However pre-processing could also destroy valuable information and therefore these methods should be applied carefully.

Researchers are often interested in finding the most relevant few wavelengths that could influence the quality evaluation of the product (ElMasry et al., 2007). Several strategies have been performed to select few wavelengths from hyperspectral data in order to design the best adapted multispectral imaging system, such as general visual inspection of the spectral curves and correlation coefficients (Keskin et al., 2004), analysis of spectral differences from the average spectrum (Liu et al., 2003), stepwise regression (Chong and Jun, 2005), orthogonal projection methods such as principal component analysis (Mehl et al., 2004; Xing and De Baerdemaeker, 2005) or covariance selection (CovSel) method (Roger et al., 2011), a priori knowledge of pigment spectral signatures and/or comparison of different spectral indexes (Merzlyak et al., 2003; Zude, 2003; Mehl et al., 2004; Lu and Peng, 2006; Lleo et al., 2011).

There are many published results on hyperspectral imaging with regard to fruit and vegetable safety (ElMasry et al., 2012). Some of them are related to the detection of sour skin (Burkholderia cepacia) in infected onions (Wang et al., 2012), fecal contamination in apples (Liu et al., 2007) and contamination by fungi in maize (Del Fiore et al., 2010). However, few applications of hyperspectral vision systems have been focused on ready to use leafy vegetables. As an example, there is a research concerning rapid detection of Escherichia coli contamination in packaged fresh spinach using hyperspectral imaging (Siripatrawan et al., 2011). This paper is a first approach to explore if hyperspectral image system is sensitive to physiological processes of aging in spinach leaves, without considering the idea of calibrating any chemical model.
Using an HIS camera raises the problem of having a good optical reference or complicated atmospheric models for obtaining the true reflectance. The present research was carried out under laboratory conditions, and this paper presents an exploitation of hyperspectral images without knowing the true reflectance.

The objective of the present work was to establish the most simple models or indexes to sense the degradation process during shelf-life of leafy vegetables. The research aims at proposing (a) the development and optimization of a hyperspectral vision system for monitoring the degradation of minimally processed spinach leaves during storage, and (b) the establishment of multivariate analysis procedures to identify and classify the damages that occur over the lifetime of the product, such as the changes of pigments and structure and incipient rost.

Models with decreasing degree of complexity are compared according to their ability for segregating spinach leaves at different quality stages. In a first step, all the wavelengths of the spectra were taken into account, computing spectral angle mapper distance to the reference spectra (SAM), in a second step partial least squares discriminant analysis models (PLS-DA) were performed, and finally the method of variable selection called CovSel (covariance selection) was implemented and a non linear index based on a subset of the selected wavelengths is proposed.

\section{Materials and methods}

\subsection{Sample collection}

Two sets of samples of spinach (Spinacia oleracea) minimally processed and packed in sealed plastic bags $(200 \mathrm{~g})$ were considered for further tests and analysis, with the goal of generating sufficient variability in the rate of deterioration. A single leaf of spinach was considered as one sample unit.

Two sets of experiments were conducted on those two sets of samples. In the first experiment, packed leafy spinach purchased from a local wholesale produce distributor was stored at $20^{\circ} \mathrm{C}$ (E-20), in order to accelerate the degradation process, and measurements were made the day of reception of samples in the laboratory (T0) and after 2 days of storage (T2). In the second experiment, the other set of samples with the same origin was left at $10^{\circ} \mathrm{C}(\mathrm{E}-10)$; the measurements were taken throughout the period of storage, beginning the day of reception (T0) and repeating the acquisition of Images 3, 6 and 9 days after (T3, T6 and T9 respectively). Twenty leaves per day of test were analyzed, and at each date, leaves coming from two different bags were considered (Table 1). Therefore, two unopened bags of spinach were used for each date. Fig. 1 shows RGB images of representative leaves of the 4 days of measurements of the $\mathrm{E}-10$ test.

\subsection{Hyperspectral imaging}

The hyperspectral vision system consisted of a push-broom EMCCD Luca-R camera (AndorTM Technology, Northern Ireland) equipped with a spectrograph Hyperspec ${ }^{\mathbb{B}}$ VNIR (spectral range: $400-1000 \mathrm{~nm}$ ). The spectral binning was configured to obtain

Table 1

Dates of measurements and number of leaves per date of measurements for E-10 and E-20.

\begin{tabular}{|c|c|c|}
\hline Experiments & $\mathrm{E}-20$ & $\mathrm{E}-10$ \\
\hline Storage temperature & $20^{\circ} \mathrm{C}$ & $10^{\circ} \mathrm{C}$ \\
\hline Dates of measurements & $\begin{array}{l}\text { - Day of reception of samples in the laboratory (T0) } \\
\text { - After } 2 \text { days of storage (T2) }\end{array}$ & $\begin{array}{l}\text { - Day of reception of samples in the laboratory (T0) } \\
\text { - After } 3 \text { days of storage (T3) } \\
\text { - After } 6 \text { days of storage (T6) } \\
\text { - After } 9 \text { days of storage (T9) }\end{array}$ \\
\hline Number of leaves per date of measurement & 20 (from two sealed packages) & 20 (from two sealed packages) \\
\hline
\end{tabular}



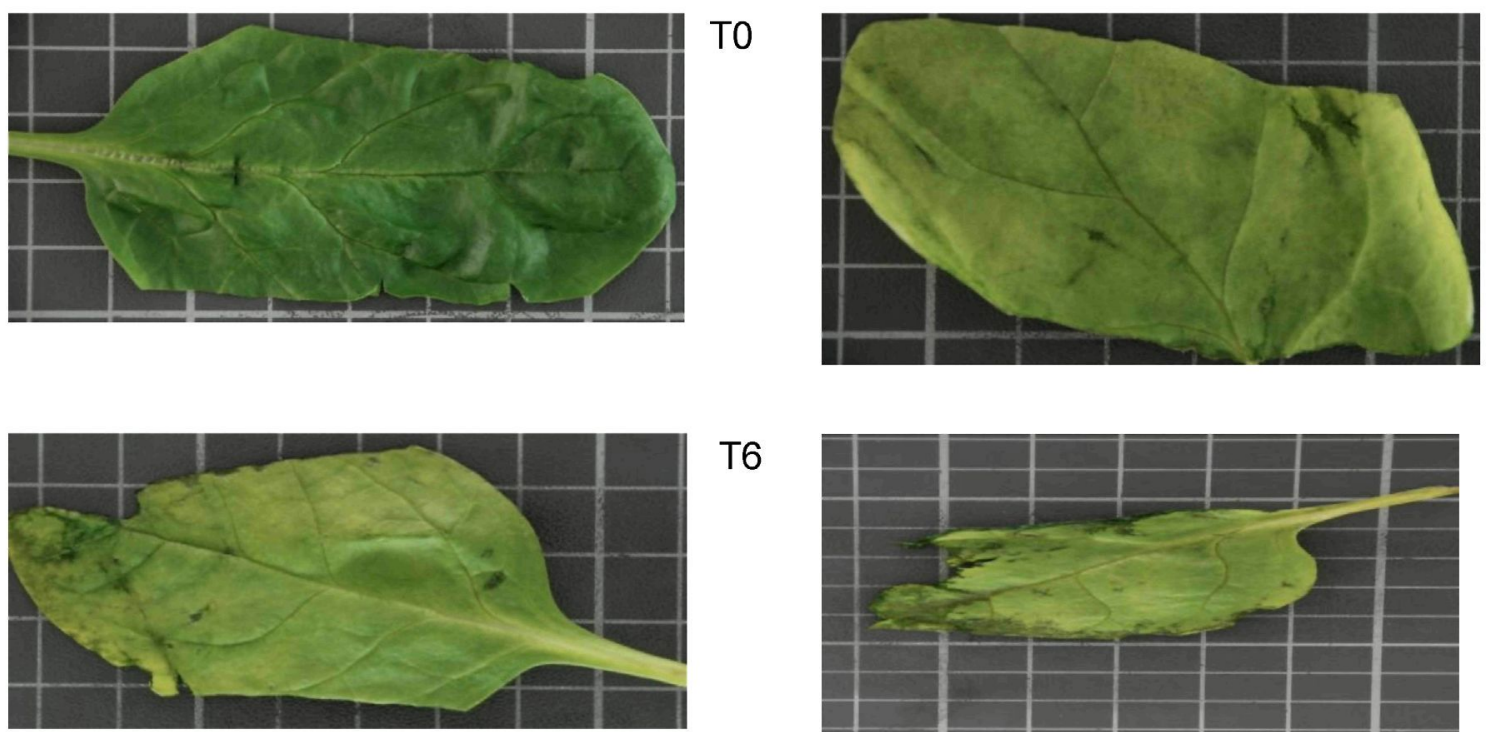

T6

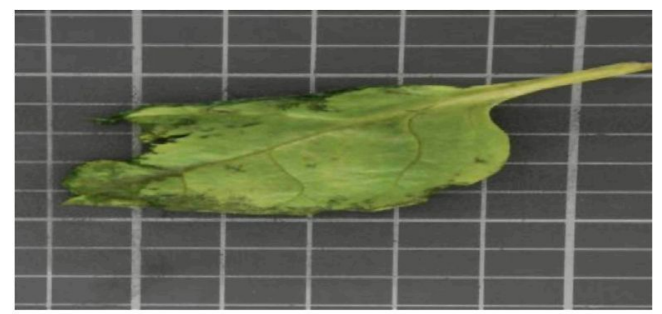

Fig. 1. Representative leaves of the E-10 set along the dates of measurements.

189 wavelengths (spectral resolution $3.17 \mathrm{~nm}$ ). The acquisition and the storage of the images were made through specific software (Headwall Hyperespec ${ }^{\circledR}$, Headwall Photonics Inc., USA). The illumination was provided by two halogens lamps. Each leaf was placed on a platform that moved (MoCo DC Motor Controller, Micos., USA) under the camera. The sample was scanned line by line according to the movement (push-broom system). The spatial resolution was $260 \mu \mathrm{m}$.

The samples were placed on a black platform to acquire the whole image of each individual leaf considering its greatest dimension direction (of the main nerve). During the acquisition of the image, the displacement of the platform was perpendicular to the direction of the main nerve, and only a width of $20 \mathrm{~mm}$ was acquired on each leaf. The surface considered on each leaf is shown on the scores images (Figs. 3 and 4 ).

The images were acquired from the leaves, with an integration time, fixed for all measurements, adapted to avoid saturation. Once the raw images were acquired, the corresponding relative reflectance hypercube was computed as follows. A reference $\left(\mathrm{Ba}_{2} \mathrm{So}_{4}\right)$ was measured, yielding $\mathrm{I}_{0}$, with an integration time also adapted to avoid saturation. The relative reflectance was then computed as the ratio of the leaf signal on the reference one. Since the integration time was not managed, the result was not meaningful in term of absolute value, but only in terms of global shape.

Leaves were distinguished from the background through the Otsu method (Otsu, 1979), a segmentation technique very commonly used in the bibliography in the case of bimodal image histograms. It was performed on the planes of $700 \mathrm{~nm}$ since they presented the greatest difference between the gray levels corresponding to leaf and to background. The Otsu algorithm for automatic threshold maximizes the between-class variance of the histogram to give the best separation. This method is based on the assumption of Gaussian (normal) distribution of both, the sample and the background histogram.

\subsection{Calibration set}

A calibration set was extracted from E-20 samples, which contained the extreme deterioration states. Three classes of degradation were identified on those images: class A, optimal quality, fresh tissue from first day (T0); class B, from non-fresh tissues belonging to samples of the second date of measurements (T2), but without visible deterioration; class $C$, regions with visible deterioration on $\mathrm{T} 2$ samples. On the hyperspectral images, areas belonging to different leaves were manually selected and their pixels assigned to one of the three defined classes. All the spectra of those regions composed the calibration set that was constituted by 3600 spectra (1200 spectra of each class). The average spectrum of each class was computed.

This calibration set was considered for the computation of SAM distance, PLS-DA model and the application of CovSel procedure.

\subsection{SAM distance}

One of the most applied strategies for material mapping is the use of similarity measures. Frequently, studies make use of a deterministic similarity measure to compare an unknown pixel spectrum with a library of reference spectra (Keshava, 2004). Spectral angle mapper (SAM) is a common distance metric, which compares an unknown pixel spectrum $t$ to each spectrum $r$ of the $K$ considered spectra of reference, and assigns $t$ to the class reference having the smallest distance. The reflectance spectra of individual pixels can be described as vectors in an $n$-dimensional space, where $n$ is the number of spectral bands. Each vector has a certain length and direction. The length of the vector represents brightness of the pixel, while the direction represents the spectral feature of the pixel. Variation in illumination mainly affects the length of the vector, while spectral variability between different spectra affects the angle between their corresponding vectors (Kruse et al., 1993). The more similar the two spectra are, the smaller the spectral angle between them. The spectral angle can have values between 0 and $\pi / 2$ and is calculated by the formula derived from the inner product of two vectors,

$$
\Theta=\cos ^{-1}\left(\frac{\sum t_{i} r_{i}}{\left(\sum t_{i}^{2} r_{i}^{2}\right)^{1 / 2}}\right),
$$

where the sum is extended to all the spectral bands (until $p$ wavelengths), $t_{i}$ and $r_{i}$ are respectively the reflectance of the actual spectrum and the reflectance of the reference spectrum at wavelength $i$. The standard spectral angle mapper (SAM), available in most image processing software packages, uses the average spectrum of each region of interest as spectrum reference (Luc et al., 2005). 
SAM distances were computed between the average spectrum of each quality class and each anonymous spectrum of the images belonging to $\mathrm{E}-20$ and $\mathrm{E}-10$ sets. From the distance values the membership degree to each class was computed in order to obtain virtual images with pixels having values between 0 and 1 . The membership degree of a pixel $p$ to the quality class $i$ is computed as

$$
\frac{1 / d_{p i}}{\sum_{i=1}^{a}\left(1 / d_{p i}\right)}
$$

where $d_{p i}$ is the distance of the spectrum of the pixel $p$ to the average spectrum of the quality class $i$, considering three classes.

Each pixel was assigned to the reference class to which it computed the maximum membership degree. Artificial images of the assignation of the pixels to the classes were computed.

\subsection{Partial least square-discriminant analysis (PLS-DA)}

The discriminant methods, which solve the issues of dimensioning and conditioning, proceed similarly to factorial regression: a classical discriminant analysis (DA) is performed on latent variables, provided either by a principal component analysis (PCA-DA), or by a PLS between the spectra and the class membership (PLS-DA). As far as regression is concerned, PLSR is generally more powerful than PCR, since the latent variable design takes into account the relationship between the spectra variables and the responses. For the same reason, in the discrimination case, PLS-DA is generally more efficient than PCA-DA (Barker and Rayens, 2003).

Partial least square discriminant analysis, PLS-DA, was applied on the calibration set ( $n=3600$ spectra, 1200 per class) between two matrices $X$ and $Y$. The spectra constituted the $X$ matrix, $n \times p$, being $n$ the total number of spectra of the calibration set, and $p$ the number of wavelengths. $Y$ matrix had $n$ rows and three columns corresponding to the three classes A, B, C of the calibration set. In the $Y$ matrix, each pixel was codified by three numbers corresponding to "membership values", one for each class, with value of 0 or 1 ; e.g., a response encoded $(010)$ means that the sample belongs to class B. Firstly, a PLS- 2 computed $k$ latent variables, which maximize the covariance between $X$ and $Y$, transforming $X$ in a $n \times k$ matrix. On this new reduced matrix, a classical linear discriminant analysis was performed to determine the most discriminant subspace. Each sample was then projected onto this subspace, yielding scores. Matlab function plsda of the PLS Toolbox (MathWorks) was used for the implementation of this analysis.

The prediction results are displayed in a confusion matrix, presenting the number of samples assigned to each class. These results allow the computation of the error of the model (Roussel et al., 2003). A cross validation process was applied, splitting the population in ten parts. The resulting errors allowed us to determine the optimal value of latent variables. Further the procedure generates discrimination vectors, which allow the projection of any anonymous individual on the space generated by these vectors obtaining their new coordinates (scores) in this space.

The model obtained from the calibration set was applied to the totality of the pixels of the hyperspectral images E-20 and $\mathrm{E}-10$, and therefore the artificial images of the scores were computed. The distance of Mahalanobis was computed between the new coordinates of the pixel (scores) and the centroid of each quality class $(A, B, C)$ of the calibration set. The Mahalanobis distance between a pixel $p$ and the centroid $Q_{i}$ of the quality class $i$ is defined as $\left[\left(p-Q_{i}\right)^{\mathrm{T}} C^{-1}\left(p-Q_{i}\right)\right]^{0.5}$, where $C$ is the covariance matrix. Each pixel of the images was assigned to the class with the maximum membership degree computed according to the distance of Mahalanobis. Artificial images of the assignation of the pixels to the classes were computed.

\subsection{CovSel computation and LEVE index}

CovSel is an algorithm which performs variable selection step by step on the basis of their global covariance with all the responses. Each variable selection is followed by the projection of the data orthogonally to the selected variable. Therefore the selected variables are independent each other as much as possible. At the exit of this procedure a set of a predetermined number $k$ of variables is generated. CovSel can be used for multi-response regression or for discrimination as well.

CovSel algorithm, described by Roger et al. (2011), was applied to the spectra of calibration set aiming at obtaining the best combination of wavelengths regarding the process of deterioration. For that, the same matrices $X, Y$ than in the mentioned above case of PLS-DA were employed for such selection. As a result, a group of $k$ wavelengths of $X$ were selected producing a smaller matrix $X^{*}$ of dimension $n$ rows and $k$ columns.

Further, a non linear index was proposed, resulting of the combination of some of the most relevant wavelengths selected by CovSel. Artificial images containing the values of this index were obtained for E-10 and E-20 sets. Analogously to the procedure explained in previous paragraphs, the distances and the membership degrees of the pixels to each quality class was computed, considering the centroid values of the index for each class. The corresponding artificial images of the assignation of pixels were also obtained for this procedure.

\subsection{Comparison between procedures}

Several procedures were carried out in order to compare the performance of each proposed procedure and its concordance in the assignation of pixels and leaves.

The artificial images of membership degrees to class $C$ obtained by the procedures proposed were studied by means of Analysis of Variance (ANOVA) in order to compare them for the detection of the degradation of the leaves along the storage period. All pixels of leaves were pulled together for the first day (first group $n=197,660$ corresponding to T0 of E-10 set) and for the last day (second group $n=182,531$ corresponding to T9 of E-10 set).

Additionally, concordance in the assignation of pixels between methods was tested taking into account the percentage of pixels assigned to the same class by the different procedures in E-20 and E- 10 sets

Finally, each leaf was assigned to the class for which it presented the maximum relative frequency (e.g., number of pixels of Class $X /$ total number of pixels of the leaf) according to SAM, PLS-DA and CovSel + LEVE index; thus three assignations (to classes A, B or C) were computed and compared for each sample.

All the analyses were made by means of routines of Matlab 7.0 (MathWorks), some of them corresponded to the packaged software (Image Analysis and PLS Toolboxes mainly), and some of them were generated to deal with specific tasks.

\section{Results and discussion}

Preliminary analysis (data not shown) showed that the best performance of the models was obtained considering raw reflectance spectra; thus no pre-processing techniques were applied to the reflectance spectra before the multivariate data analysis.

Fig. 2 a shows the average spectra of the 3 classes A, B, C calculated on the calibration set ( $n=1200$ for each class). The reflectance spectrum corresponding to class $C$ presents a general decrease in the whole range from 450 till $1000 \mathrm{~nm}$, being particularly high in the region of NIR from 700 till $1000 \mathrm{~nm}$. In addition, the slope of the spectrum between 710 and $900 \mathrm{~nm}$ is increasing from class 
a

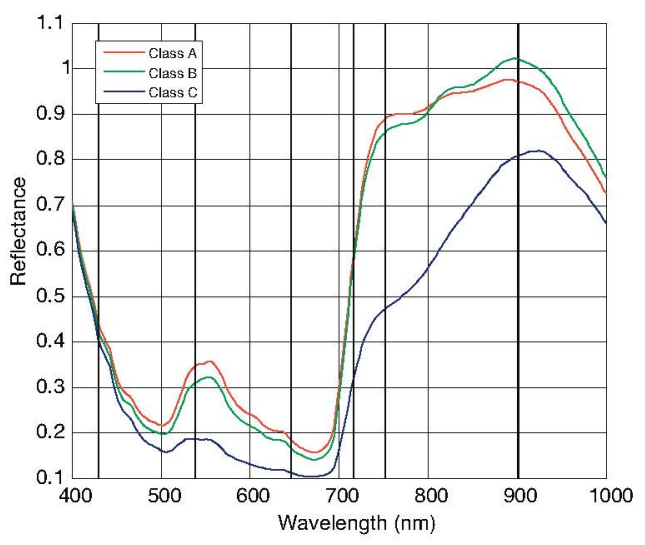

C

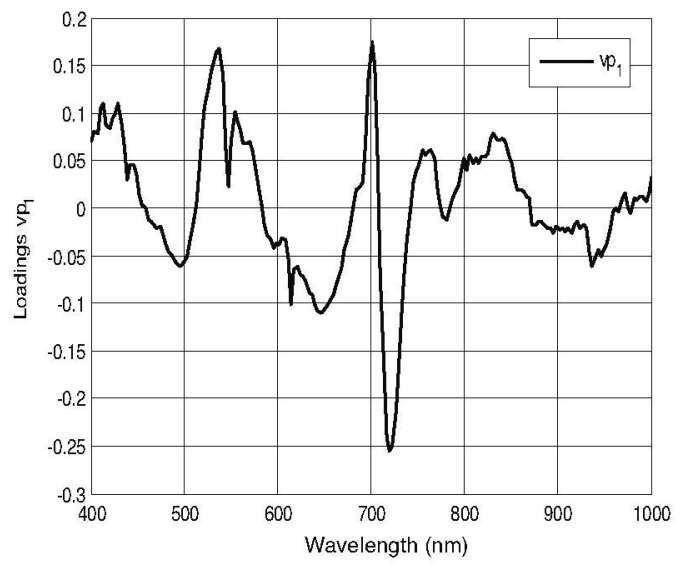

b

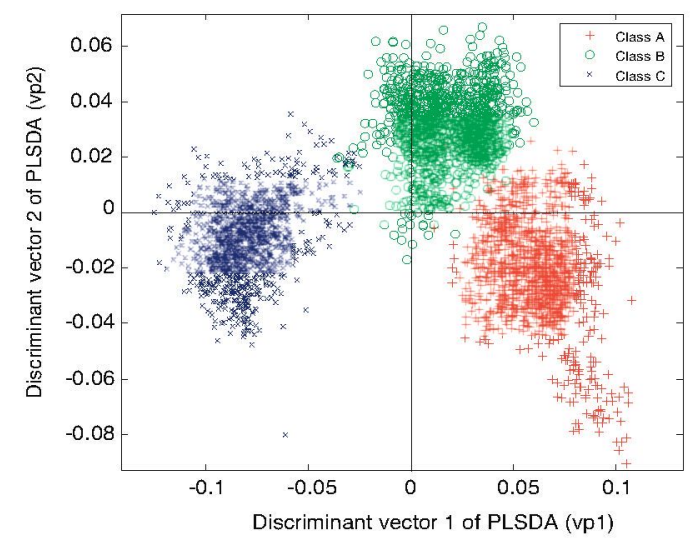

d

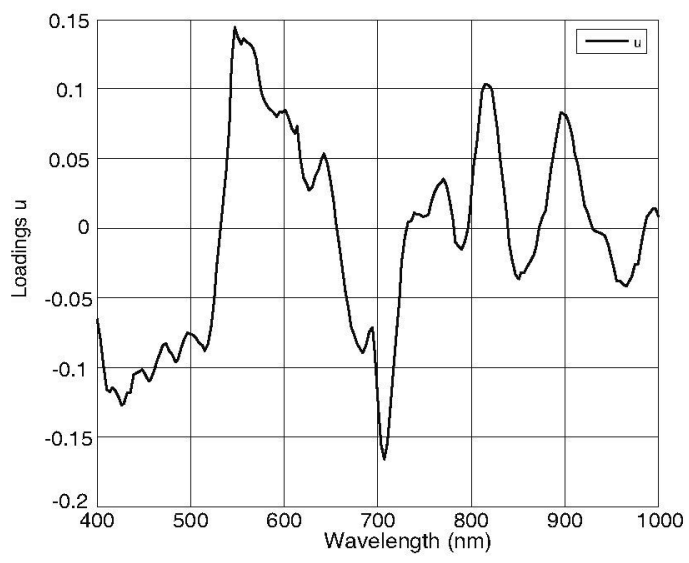

Fig. 2. (a) Average spectra of the 3 classes A, B and C ( $n=3600$ spectra in total); vertical lines indicate the 6 wavelengths selected by CovSel; (b) scores of the 3 classes calculated by the PLS-DA on the learning set; (c) first discriminant vector (vp1); (d) residual of the second discriminant vector to the first one ( $u$ ).

A to class $\mathrm{C}$, this change being especially relevant for the most deteriorated samples (class $C$ ). Regarding the VIS range of the spectra, a general decrease in reflectance values from the soundest to the most deteriorated pixels was observed. The spoilage in these leaves induced spectral changes different from the ones caused by aging and senescence typically described in leaves by remote sensing methods (Asner, 1998; Liy et al., 2010). These authors stated that aging of vegetation induces a decrease in water content and in some foliar pigments such as chlorophyll, although carotenoids may increase during cold storage. As a result, global reflectance increases in the visible and near-infrared range. The high decrease in global reflectance in the spectra of class $C$ could indicate that the main effect of the degradation is not water loss, suggesting that the spoilage suffered by packed leaves is different from the typical deterioration observed in vegetation by remote sensing. In fact, the deteriorated tissues observed in packed leaves seemed to be crushed and moist(Fig. 1, treatment T9), whereas in remote sensing the areas affected by deterioration show yellowness, loss of water, chlorophyll and some other pigments, but not a moist or crushed aspect.

Jacquemoud and Baret (1990) stated that the interaction of light with plant leaves depends on the chemical and physical characteristics of the tissues. Absorption is essentially a function of changes on molecules (chlorophyll a and b, carotenoids, water, etc.) and is responsible for the absorption bands that appear as valleys because we used reflectance spectrum. The refractive index discontinuities within tissue induce scattering which acts on the global trend of the reflectance spectrum. The internal structure of the leaves affects the scattering of the light, therefore it modulates the reflectance of the whole spectrum, this effect being clearer where the absorption is lower, especially in NIR. Therefore, the changes that occurred in the scattering are caused by structural changes in the leaves during cold storage, which could explain why the best models are obtained when no spectra pre-processing was carried out.

The main deterioration effect in the present study is probably the destruction of the internal structure of the tissue (wall cells) inducing an increase in free water in the intercellular space. It could be supposed that refractive index discontinuities decrease within the leaves, leading to a reduction of the light scattering and consequently a decrease in the global reflectance of the spectra. This decrease in reflectance was observed on class $C$ spectra, being especially pronounced in the NIR region (above $700 \mathrm{~nm}$ ) according to Jacquemoud and Baret (1990). Additionally the presence of free water facilitates deeper penetration of the light in the tissue, increasing the effective path length and making more probable the absorption of the light during its travel by the pigments, which decreases the reflectance, mostly in the visible range. Around the red edge $(670-720 \mathrm{~nm})$ both phenomena occurred: (a) a general decrease of global reflectance in NIR region, and (b) a specific decrease in reflectance at the red edge induced by the absorption of the chlorophyll. The high slope at the NIR region $(700-900 \mathrm{~nm})$ in the average spectrum of class C (Fig. 2a) could be caused by 
differences in the intensities of these two phenomena in the red edge range and in the rest of the NIR range where the effect of the absorption could be considered negligible. The average spectrum of class B showed an intermediate stage that could denote an incipient phase of the degradation of leaf structure.

\subsection{PLS-DA/CovSel}

A total of 7 latent variables were chosen for the PLS-DA by means of a cross-validation procedure (Geisser, 1993). In addition, the score images showed less noise with 7 than with 8 latent variables. In images obtained using 8 latent variables, structure details of the leaves, such as veins, were not clearly identified (image not shown).

Fig. 2b shows the score plot of the calibration set. Each point of this graph corresponds to the projection of one spectrum of this set onto the 2-dimensional discriminant space spanned by the discriminant vectors of the PLS-DA $\left(\mathrm{vp}_{1}, \mathrm{vp}_{2}\right)$. Thus, the abscissa and the ordinates of the scores can be interpreted with regard to the combination of spectra features (peaks, slopes, etc.) and the shape of the discriminant vectors. However, PLS-DA discriminant vectors are normalized but not orthogonal; in our case they are almost colinear, making their interpretation difficult. Trying to improve the interpretation of the discriminant vectors, a new vector $(u)$ was computed by orthogonalizing $\mathrm{vp}_{2}$ against $\mathrm{vp}_{1}: u=\left(I-\mathrm{vp}_{1}^{\mathrm{T}} \mathrm{vp}_{1}\right) \mathrm{vp}_{2}$. Fig. $2 \mathrm{c}$ and $\mathrm{d}$ presents the two vectors $\mathrm{vp}_{1}$ and $u$, respectively.

The score plot of Fig. $2 \mathrm{~b}$ shows that $\mathrm{vp}_{1}$ clearly orders the 3 classes from $C$ (negative scores) to A (positive scores), through $B$ (null and little positive scores). On this axis, classes $A$ and $B$ are overlapping. The discriminant vector $\mathrm{vp}_{1}$ (Fig. $2 \mathrm{c}$ ) shows three main features that explain that ordering: a large positive peak around $550 \mathrm{~nm}$ contributes positively to the score for spectra presenting a high reflectance in this zone, as those of classes $\mathrm{A}$ and $\mathrm{B}$, meaning that the leaves are less and less green while aging; a sharp positive peak at $700 \mathrm{~nm}$ computes a part of the scores which corresponds to the position of the red edge, which collapse when the leaves age; the combination of a negative peak at $720 \mathrm{~nm}$ and a positive zone around $760 \mathrm{~nm}$ computes the slope of the red edge, which decreases when the leaves age.

The second axis of the score plot (Fig. 2b) discriminates the low scores of classes $\mathrm{A}$ and $\mathrm{C}$ from the high scores of the class $\mathrm{B}$. The vector $u$ (Fig. 2d), which represents the part of vp2 not included in vp1, shows also three main features: a negative zone between 400 and $530 \mathrm{~nm}$ combined with a positive one between 530 and $660 \mathrm{~nm}$ realizes a colorimetric balance between blue/green and yellow/red color of the leaves, denoting changes in pigment contents; a series of peaks and valleys in the NIR region, between 800 and $1000 \mathrm{~nm}$. This shape cannot be finely analyzed, but may be due to subtle changes in chemicals. Indeed, this spectral zone is affected by sugars, proteins and water (Osborne and Fearn, 1986); a negative peak at $707 \mathrm{~nm}$, which cannot be directly interpreted relatively to score plot.

The six wavelengths chosen by the CovSel algorithm were: 752, 902, 538, 717, 430 and $647 \mathrm{~nm}$, as reported in Fig. 2a. With the perspective of a multispectral approach, a new non linear index was tested based on the most relevant wavelengths selected by CovSel and on the loadings of the first discriminant vector of PLS-DA. Leafy Vegetable Evolution index (LEVE index) focuses on two spectral regions: NIR region $(750-900 \mathrm{~nm})$ and VIS region (506-614 nm). LEVE index is defined by the expression $\left(\left(R_{900}-R_{750}\right) /\left(R_{900}+R_{750}\right)\right) /\left(\left(R_{519}+R_{646}\right) / R_{538}\right)$, where $R_{x}$ corresponds to the relative reflectance at $x \mathrm{~nm}$.

The numerator of LEVE index located at NIR region computed a kind of normalized slope between 750 and $900 \mathrm{~nm}$, including the two first wavelengths selected by CovSel. The denominator located at VIS region (including two of the wavelengths selected by CovSel) was an approximation to the minus normalized second derivative at $538 \mathrm{~nm}$ according to the proposal by Lleo et al. (2011). LEVE index increased from class A to class $C$.

\subsection{Virtual images of membership degree}

In Figs. 3 and 4, the virtual images of membership degree to class $C$ are showed for the three described classification procedures (i.e. based on SAM, PLS-DA and CovSel plus LEVE index) applied on samples of set E-20 and E-10 respectively. All the procedures accordingly identified the regions belonging to class $C$. More accentuated degradation could be observed on samples from set E-20, which is concordant with the storage conditions. Inside each leaf pixels can be observed with a very different rate of deterioration. In addition, leaves belonging to the same storage conditions presented different rates of degradation. This fact is especially evident on the second date (second row of images of Fig. 4) of E-20, where the third, fourth and sixth leaves (from left of the row) contained little regions close to class $\mathrm{C}$, whereas the last five leaves showed patches of very deteriorated pixels. Regarding E-10, on the third line at Fig. 5 (third measurement date), four leaves presented an appearance similar to the corresponding to the first date of measurements. That suggests that the degradation rate of the leaves is very heterogeneous inside commercial batches, which could be explained by the original condition of the vegetal material on harvest and postharvest chain (Medina et al., 2012).

For ANOVA computation the membership degrees to class $C$ obtained by the three methods was considered, comparing the first date of measurements of E-10 spectra with the last date ( $n=197,660$ pixels for first date; $n=182,531$ pixels for last date). All methods sense the degradation with a better performance for PLS-DA ( $F$ value 582,705 at $p$ level 0 ), which could be expected taking into account that this model includes all the wavelengths and it is based on the covariance matrix between the spectra $(X)$ and the visual assignation to the deterioration classes $A, B$ and $C(Y)$. Similar $F$ values were obtained for PLS-DA and LEVE index, 333,099 and 320,215 respectively.

\subsection{Virtual images of assignation to quality class}

As mentioned before all pixels of the leaves were assigned to their closest class according to membership degree; the assignation was to the class with highest membership degree. Fig. 5 shows, such as example, the virtual images for assignation to the classes according to LEVE index. Similar pattern of distribution of regions in the three artificial images of pixels classification was observed in calibration (E-20) and validation (E-10) sets of leaves (data not shown). The analysis of this observation was performed quantifying the percentage of pixels with the same assignation for the different procedures (Table 2 ).

The assignation of the pixels to one of the three classes showed high concordance when comparing the three methods of analysis. The highest levels of concordance occurred between the

Table 2

Percentage of concordance between methods in the assignation of the pixels of leaves to the classes $\mathrm{A}, \mathrm{B}$ and $\mathrm{C}$ for $\mathrm{E}-20$ and $\mathrm{E}-10$ samples.

\begin{tabular}{lccc}
\hline & SAM $(\%)$ & PLS-DA $(\%)$ & LEVE index $(\%)$ \\
\hline E-20 & & & \\
SAM & 100 & 77 & 93 \\
PLS-DA & 77 & 100 & 76 \\
LEVE index & 93 & 76 & 100 \\
E-10 & & & \\
SAM & 100 & 80 & 87 \\
PLS-DA & 80 & 100 & 75 \\
LEVE index & 87 & 75 & 100 \\
\hline
\end{tabular}




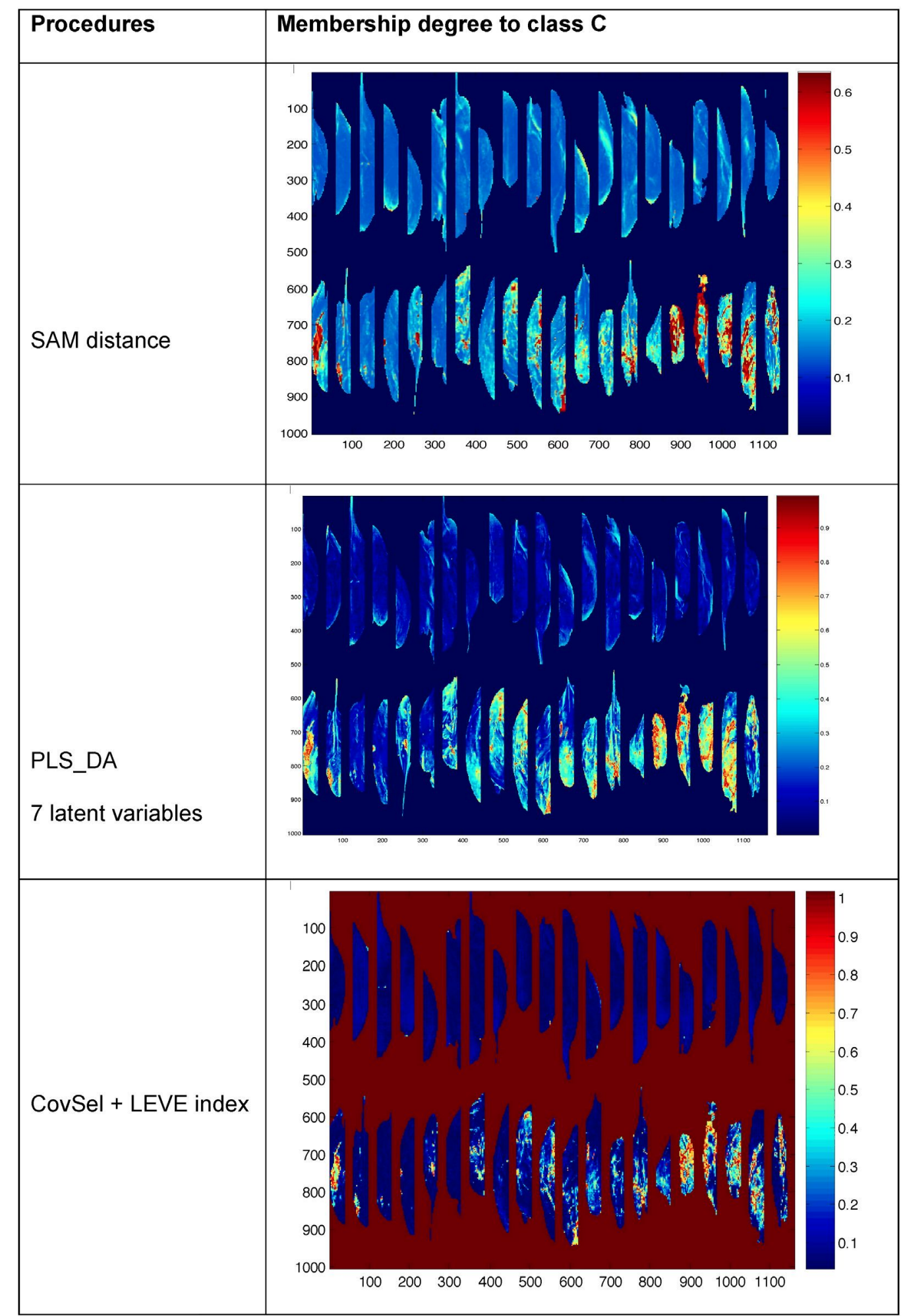

Fig. 3. Visualization of the membership degree to class $C$, in E-20 samples, for the different proposed procedures (each row of the table). Redder pixels are closest to class $C$ (more deteriorated areas) whereas deep blue pixels are far from class $\mathrm{C}$ (healthy tissues). Each image contains the leaves corresponding to first date, first row, ant last date of measurements, second row. (For interpretation of the references to color in this figure legend, the reader is referred to the web version of the article.)

assignations based on SAM distance and on LEVE index (93\% and $87 \%$ for E-20 and E-10 sets respectively), whereas the most discrepant assignations corresponded to the comparison between PLS-DA and LEVE index (76\% and 75\% for E-20 and E-10 sets). Consequently, LEVE index could be employed instead of PLS-DA or SAM distance, presenting the advantage of using only 5 wavelengths and not all the spectra, which could be integrated into a simpler multispectral vision system.

In addition, classification of leaves into the class in which the relative frequency of pixels is the highest reinforced the fact of 


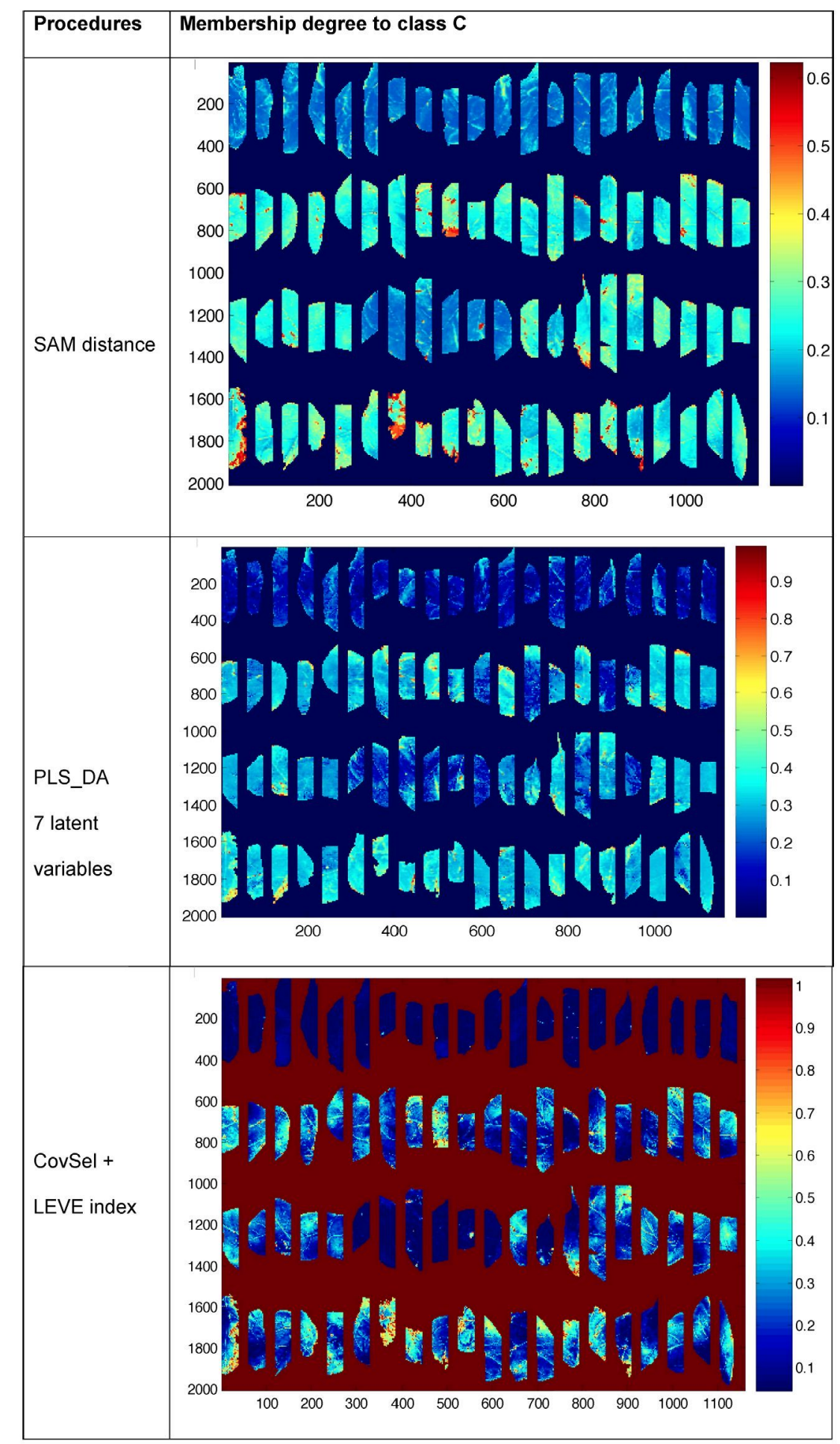

Fig. 4. Visualization of the membership degree to class $C$, in E-10 samples, for the different proposed procedures. Redder pixels are closest to class $\mathrm{C}$ (more deteriorated areas) whereas deep blue pixels are far from class $C$ (healthy tissues). Each image contains the leaves corresponding to the four dates of measurements: each row corresponds to one of the dates of measurements. (For interpretation of the references to color in this figure legend, the reader is referred to the web version of the article.)

concordance between methods (Table 3 ): the $82.5 \%$ and the $92.5 \%$ of leaves of $\mathrm{E}-20$ and $\mathrm{E}-10$ respectively, were classified in the same category by the three methods. The highest level of coincidence was achieved between SAM distance and LEVE index with the 95\% (E20 ) and $97.5 \%(E-10)$ of the leaves assigned to the same quality class.
Regarding the discrepancies, LEVE index assigned higher membership degree to class $C$ which allowed segregating better between class $C$ and class B. Fig. 6 presents the images of membership degree to class $C$ of one leave of $E-10$ set assigned to class $B$ according to SAM distance (left) and to class C according to LEVE index (right). 


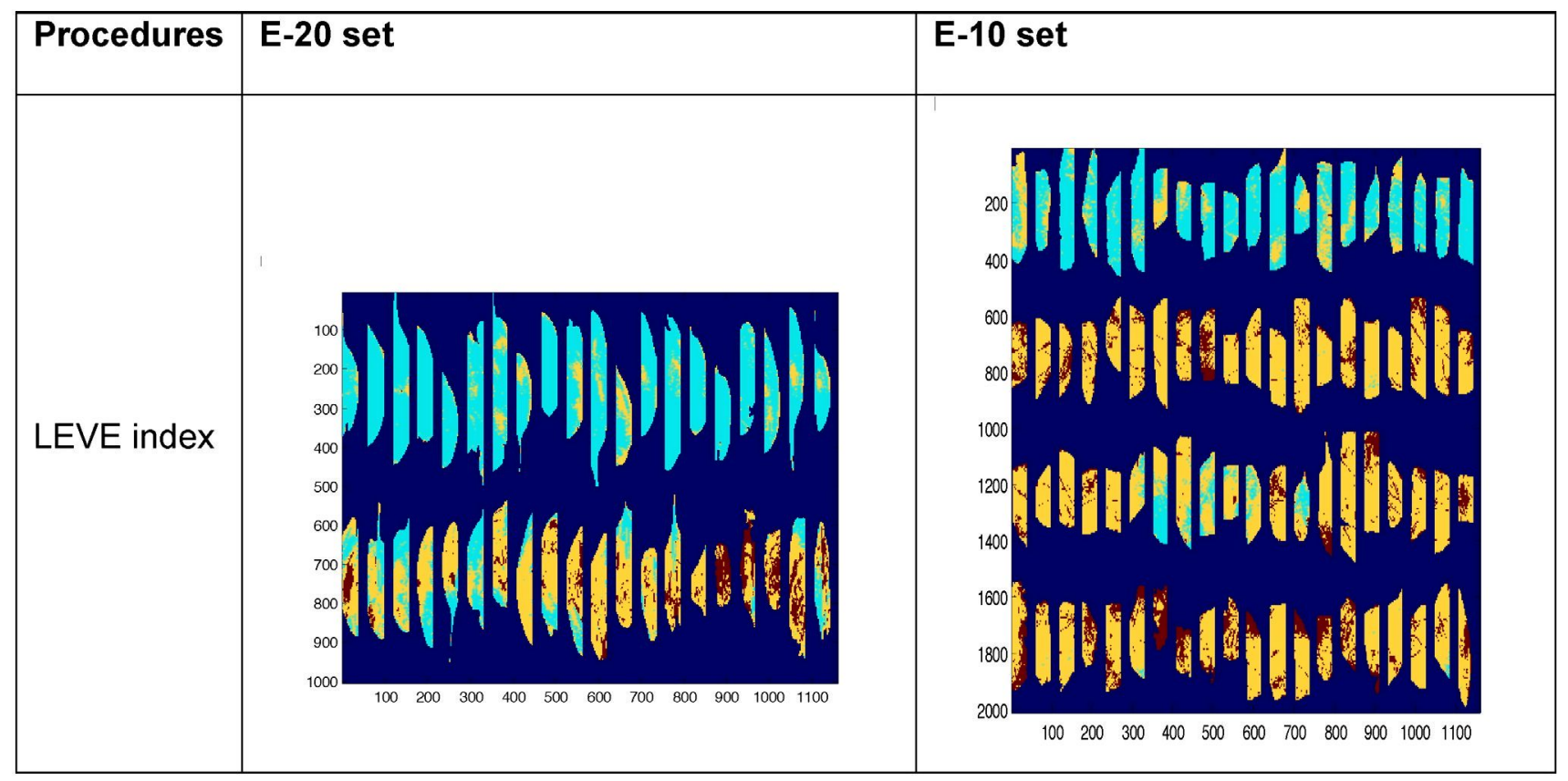

Fig. 5. Visualization of the assignation of each pixel to one of the three quality classes considering the maximum membership degree. Blue color corresponds to class $A$, orange color to class $\mathrm{B}$ and color brown to class C. (For interpretation of the references to color in this figure legend, the reader is referred to the web version of the article.)

Table 3

Percentage of concordance between methods in the assignation of the leaves to the three classes $\mathrm{A}, \mathrm{B}$ and $\mathrm{C}$ for $\mathrm{E}-20$ and $\mathrm{E}-10$ samples.

\begin{tabular}{lccc}
\hline & SAM $(\%)$ & PLS-DA (\%) & LEVE index (\%) \\
\hline E-20 & & & \\
SAM & 100 & 85 & 95 \\
PLS-DA & 85 & 100 & 85 \\
LEVE index & 95 & 85 & 100 \\
E-10 & & & \\
SAM & 100 & 93.75 & 97.5 \\
PLS-DA & 93.75 & 100 & 93.75 \\
LEVE index & 97.5 & 93.75 & 100 \\
\hline
\end{tabular}

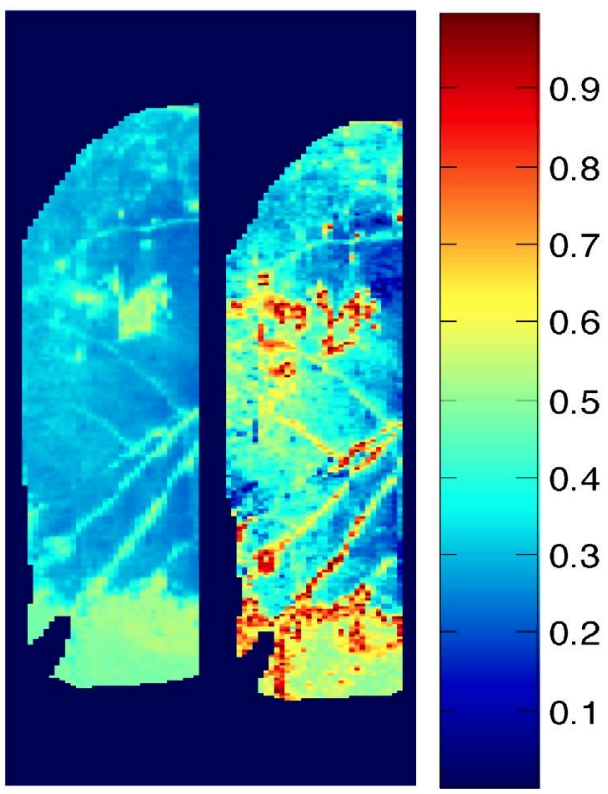

Fig. 6. Images of membership degree to class $C$ of one leaf of $E-10$ set assigned to class B according to SAM distance (left) and to class Caccording to LEVE index (right).
It suggests that if the identification of all the possible problematic leaves is crucial, it might be better to use LEVE index instead of SAM distance.

\section{Conclusions}

The main differences observed in the reflectance spectra over storage time was an overall decline in the intensity of VIS and NIR ranges and an increase in the slope of between 710 and $900 \mathrm{~nm}$, which was particularly pronounced for the most deteriorated quality category. A hypothesis has been formulated for the explanation of these effects: the destruction of the internal structure of tissue cause an increase in free water content and consequently the decrease of the refractive index discontinuities within the leaves and the reflectance of the spectra. Furthermore, the same fact also facilitates the deeper penetration of light increasing the probability of being absorbed by pigments in the VIS range, which also decrease the reflectance. The differences in the intensities of these two phenomena (increase in free water content and increase in absorption of the light by pigments) in the red edge could explain the high slope at the NIR region in the average spectrum of the most deteriorated class.

The tests performed showed the ability in discriminating between different storage periods of virtual images resulting from the application of three analytical techniques (SAM, PLS-DA, CovSel and non linear index) to the hyperspectral images.

Due to the high intra-date and intra-leaf variability, the study of a first calibration set composed according to dates was unsuccessful. Therefore, a calibration population (3600 spectra) was defined by a supervised selection visually of pixels according to their degradation stage.

The implementation of different multivariate techniques on the calibration sub-population generated classification models in three categories that responded to the main states of degradation, followed by the product in the tests. The projection of the hyperspectral images onto the generated subspaces and the assignation of each pixel to one of the defined categories, allowed the identification of regions with different states of degradation in the leaves. 
The system made it possible to determine the percentages of the areas of these regions and to establish decision rules about the overall quality of the leaf, which is a clear advantage compared to the spectrophotometric methods that analyze a small area of the sample.

The method CovSel, dedicated to the problem of variable selection for highly multivariate data, allowed the identification of the most relevant (and independent) 10 wavelengths, which were the starting point for the definition of a non linear index, LEVE index, combining 5 of the 10 wavelengths selected by CovSel, achieving similar results that SAM distance analysis and PLS-DA, these last two procedures using the complete spectra. More than the 95\% of the leaves were classified into the same quality class by SAM distance and by LEVE index.

\section{Acknowledgements}

The funding of this work has been covered by the MICINN with the project Multihort (AGL2008-05666-C02-01) and by the Technical University of Madrid with de project Durasfrut II (AL11$\mathrm{P}(\mathrm{I}+\mathrm{D})-06)$. LPF-TAGRALIA is part of the CEI Moncloa Campus of Excellence, UPM-UCM.

\section{References}

Asner, G.P., 1998. Biophysical and biochemical sources of variability in canopy reflectance. Remote Sens. Environ. 64, 234-253.

Barker, M., Rayens, W., 2003. Partial least squares for discrimination. J. Chemometr. $17,166-173$

Chao, K., Chen, Y.R., Hruschka, W.R., Park, B., 2001. Chicken heart disease characterization by multi-spectral imaging. Appl. Eng. Agric. 17, 99-106.

Chong, I.G., Jun, C.H., 2005. Performance of some variable selection methods when multicollinearity is present. Chemom. Intell. Lab. Syst. 78, 103-112

Del Fiore, A., Reverberi, M., Ricelli, A., Pinzari, F., Serranti, S., Fabbri, A.A., Bonifazi, G., Fanelli, $C_{n}$ 2010. Early detection of toxigenic fungi on maize by hyperspectral imaging analysis. Int. J. Food Microbiol. 144, 64-71.

EIMasry, G., Sun, D.W., 2010. Principles of hyperspectral imaging technology. In: Sun, D.W. (Ed.), Hyperspectral Imaging for Food Quality Analysis and Control. Academic Press, San Diego, pp. 3-43.

ElMasry, G., Wang, N., ElSayed, A., Ngadi, M., 2007. Hyperspectral imaging for nondestructive determination of some quality attributes for strawberry. J. Food Eng. $81,98-107$.

EIMasry, G., Kamruzzaman, M., Sun, D.W., Allen, P., 2012. Principles and applications of hyperspectral imaging in quality evaluation of agro-food products. A review. Crit. Rev. Food Sci. 52, 999-1023.

Fearn, T., Riccioli, C., Garrido-Varo, A., Guerrero-Ginel, J.E., 2009. On the geometry of SNV and MSC. Chemom. Intell. Lab. Syst. 96, 22-26.

Fernandez Pierna, J.A., Vermeulen, P., Dardenne, P., Baeten, V., 2010. Integration of Chemometric Tools in Hyperspectral Imaging Data: Contaminant Detection. International Association for Spectral Imaging. IASIM-10, Dublin, Ireland.

Geisser, S., 1993. Predictive Inference: An Introduction. Chapman and Hall, New York, NY.

Gowen, A.A., O’Donnell, C.P., Cullen, P.J., Downey, G., Frias, J.M., 2007. Hyperspectral imaging-an emerging process analytical tool for food quality and safety control. Trends Food Sci. Technol. 18, 590-598.

Hernandez-Sanchez, N., Lurol, S., Roger, J.M., Bellon-Maurel, V., 2003. Robustness of models bases on NIR spectra for sugar content prediction in apples. J. Near Infrared Spectrosc. 11,97-107.

Jacquemoud, S., Baret, F., 1990. Prospect - a model of leaf optical properties spectra. Remote Sens. Environ. 34, 75-91.
Keshava, N., 2004. Distance metrics and band selection in hyperspectral processing with application to material identification and spectral libraries. IEEE Trans. Geosci. Remote 42, 1552-1565.

Keskin, M., Dodd, R.B., Han, Y.J., Khalilian, A., 2004. Assessing nitrogen content of golf course turfgrass clipping using spectral reflectance. Appl. Eng. Agric. 20 , $851-860$.

Kruse, F., Lekoff, A., Boardman, J., Heidebrecht, K., Shapiro, A., Barloon, P., Goetz, A., 1993. The spectral image processing system (SIPS) - interactive visualization analysis of imaging spectrometer data. Remote Sens. Environ. 44, 145-163.

Lee, K.J., Kang, S., Kim, M.S., Noh, S.H., 2005. Hyperspectral imaging for detecting defect on apples. In: ASAE (Ed.), ASAE Annual International Meeting. ASAE, Tampa, FL, USA.

Liu, Y., Windham, W.R., Lawrence, K.C., Park, B., 2003. Simple algorithms for the classification of visible/near-infrared and hyperspectral imaging spectra of chicken skins, feces, and fecal contaminated skins. Appl. Spectrosc. 57, 1609-1612.

Liu, Y.L., Chen, Y.R., Kim, M.S., Chan, D.E., Lefcourt, A.M., 2007. Development of simple algorithms for the detection of fecal contaminants on apples from visible/near infrared hyperspectral reflectance imaging. J. Food Eng. 81, 412-418.

Liy, Z.Y., Shi, J.J. Wang, D.C., Huang, J.F., 2010. Discrimination and spectral response characteristic of stress leaves infected by rice Aphelenchoides besseyi Christie. Spectrosc. Spectral Anal. 30, 710-714.

Lleo, L., Roger, J.M., Herrero-Langreo, A., Diezma-Iglesias, B., Barreiro, P., 2011. Comparison of multispectral indexes extracted from hyperspectral images for the assessment of fruit ripening. J. Food Eng. 104, 612-620.

Lu, R., Peng, Y., 2006. Hyperspectral scattering for assessing peach fruit firmness. Biosyst. Eng. 93, 161-171.

Luc, B., Deronde, B., Kempeneers, P., Debruyn, W., Provoost, S., 2005. Optimized Spectral Angel Mapper classification of spatially heterogeneous dynamic dune vegetation, a case study along the Belgian coastline. In: ISPMSRS (Ed.), The 9th International Symposium on Physical Measurements and Signatures in Remote Sensing.

Martín-Belloso, O., Soliva-Fortuny, R., 2011. Advances in Fresh-cut Fruits and Vegetables Processing. CRC Press Taylor \& Francis Group.

Medina, M.S., Tudela, J.A., Marín, A., Allende, A., Gil, M.I., 2012. Short postharvest storage under low relat ive humidity improves quality and shelf life of minimally processed baby spinach (Spinacia oleracea L.). Postharvest Biol. Technol. 67, 1-9.

Mehl, P.M. Chen, Y,-R, Kim, M.S, Chan, D.E, 2004. Development of hyperspectral imaging technique for the detection of apple surface defects and cont aminations. J. Food Eng. 61, 67-81.

Merzlyak, M.N., Solovchenko, A.E., Gitelson, A.A., 2003. Reflectance spectral features and non-destructive est imation of chlorophyll, carotenoid and anthocyanin content in apple fruit. Postharvest Biol. Technol. 27, 197-211.

Osborne, B.G., Fearn, T., 1986. Near Infrared Spectroscopy in Food Analysis. Longman Scientific and Technical, Harlow, UK

Otsu, N., 1979. A threshold selection method from gray-level histogramas. IEEE Trans. Syst. Man. Cybern. 9, 62-66.

Rinnan, A. Van Den Berf, F, Engelsen, S.B., 2009. Review of the most common pre-processing techniques for near-infrared spectra. Trends Anal. Chem. 28, 1201-1222.

Roger, J.M., Palagos, B., Bertrand, D., Fernandez-Ahumada, E., 2011. CovSel: variable selection for highly multivariate and multi-response calibration, application to IR spectroscopy. Chemom. Intell. Lab. Syst. 106, 216-223.

Roussel, S., Bellon-Maurel, V., Roger, J.M., Grenier, P., 2003. Authenticating white grape must variety with classification models based on aroma sensors, FT-IR and UV spectrometry. J. Food Eng. 60, 407-419.

Siripatrawan, U, Makino, Y. Kawagoe, Y, Oshita, S, 2011. Rapid detection of Escherichia coli contamination in packaged fresh spinach using hyperspectral imaging. Talanta $85,276-281$

Wang, W., Li, C., Tollner, E.W., Gitaitis, R.D., Rains, G.C., 2012. Shortwave infrared hyperspectral imaging for detecting sour skin (Burkholderia cepacia)-infected onions. J. Food Eng. 109, 38-48.

Xing, J., De Baerdemaeker, J., 2005. Bruise detection on 'Jonagold' apples using hyperspectral imaging. Postharvest Biol. Technol. 37, 152-162.

Zeaiter, M., Roger, J.M., Bellon-Maurel, V., 2005. Robustness of models developed by multivariate calibration. Part II. The influence of pre-processing methods. Trends Anal. Chem. 24, 437-445.

Zude, M., 2003. Comparison of indices and multivariate models to non-destructively predict the fruit chlorophyll by means of visible spect rometry in apple fruit. Anal. Chim. Acta 481, 119-126. 